\title{
A prospective study of the causes of notably raised aspartate aminotransferase of liver origin
}

\author{
M W Whitehead, N D Hawkes, I Hainsworth, J G C Kingham
}

\begin{abstract}
Background and aims-To ascertain the causes of raised aspartate aminotransferase (AST) presumed to be of hepatic origin in two hospitals and the local community served by a centralised biochemistry laboratory.

Methods-From June 1996 to February 1997 all patients with AST greater than 400 U/1 were identified by the biochemistry laboratory; the patients' clinical records were studied to determine the diagnosis, the clinical outcome, and whether the raised AST and its significance had been noted.

Results-A total of 137 patients with a hepatic cause for the raised AST were found. The cause of the raised AST was hepatic ischaemia/hypoxia in 68 , pancreatobiliary disease in 33, primary hepatocellular disease in 23 , hepatic malignancy in five, and hepatic haematoma in one. In seven patients the diagnosis was unclear. The overall mortality was high $(22 \%)$ with the highest mortality in the hepatic ischaemia group $(37 \%)$. The recording and interpretation of the causes of raised AST was poor with only $48 \%$ having the correct diagnosis. In $38 \%$ the raised AST was apparently not noticed by the attending clinicians.

Conclusions-The commonest cause of a hepatitis like biochemical picture was hepatic hypoxia (50\%) followed by pancreatobiliary disease (24\%). Drug induced hepatic necrosis $(8.8 \%)$ was uncommon and viral hepatitis was rare $(3.6 \%)$. AST concentrations returned towards normal most rapidly in patients with hepatic hypoxia and calculous biliary obstruction. Hepatitis, viral or otherwise, is an uncommon cause of a typical hepatitic biochemical result in this community.

(Gut 1999;45:129-133)
\end{abstract}

Keywords: aspartate aminotransferase; hepatic hypoxia; ischaemia; hepatitis

Aspartate aminotransferase (AST) is a mitochondrial enzyme involved in the transfer of an amino group from a 2 -amino- to a 2 -oxoacid found in the heart, liver, skeletal muscle, and kidney and is normally present in plasma. ${ }^{1}$ The serum concentrations are raised when any of these tissues are damaged and are clinically useful in the diagnosis of hepatobiliary disease, myocardial infarction, skeletal muscle necrosis, and pulmonary infarction.
A notable increase in AST was shown in patients with viral hepatitis over 40 years ago by De Ritis and Coltorti ${ }^{2}$ and since then this has been the standard biochemical indicator of hepatocyte damage. In acute viral hepatitis 20-30-fold elevations of AST are common in the early phase with a gradual return to normal over a few weeks if recovery is uneventful. It was soon shown that elevations of AST were seen in a wide range of hepatic disorders, ${ }^{3}$ so an abnormal result is of limited value in differential diagnosis. As a general rule elevations of AST greater than tenfold (greater than 400 $\mathrm{U} / 1$ ), point towards hepatocellular damage, while in obstructive jaundice the AST concentration is usually below $400 \mathrm{U} / 1 .{ }^{4}$ Very high AST concentrations (greater than $1000 \mathrm{U} / \mathrm{l}$ ) are characteristic of viral hepatitis or drug toxicity and are held to be of great diagnostic value. ${ }^{5}$ Such very high concentrations are also reported in circulatory collapse and occasionally in extrahepatic obstruction which may lead to an erroneous diagnosis of acute hepatitis. ${ }^{6}$ Raised AST concentrations, generally below $400 \mathrm{U} / 1$, are also seen in intrahepatic and extrahepatic cholestasis, in cirrhosis, and in primary and secondary liver tumours. ${ }^{4}$

The aim of this study was to investigate the causes of a notable elevation of AST of presumed liver origin in a prospective manner in ordinary clinical practice as seen in two large district hospitals and the community they serve. We wished to show whether standard teaching on the causes of this biochemical picture held true, and to look at the natural history of the various causes we identified. We also observed how clinicians interpret the liver function tests (LFT) of patients under their care.

\section{Methods}

In a nine month period, June 1996 to February 1997, a prospective study of all patients with an AST concentration of greater than ten times normal (greater than $400 \mathrm{U} / \mathrm{l}$ ) was carried out. The raised AST values were identified by a centralised biochemistry department. AST was measured using the IFCC method run on a Bayer 48 analyser. ${ }^{8}$

The biochemistry department serves the three district general hospitals of West Glamorgan (two in Swansea and one in Neath) and the community. For practical reasons we only studied patients from the two Swansea hospitals and the population they serve. This population is defined by the Unitary Authority of

Abbreviations used in this paper: AST, aspartate aminotransferase; LFT, liver function tests. 
Table 1 Causes of aspartate aminotransferase greater than $400 \mathrm{U} / \mathrm{l}$, showing diagnostic groups with subdivisions of these groups and the number of patients in each

\begin{tabular}{lllll}
\hline Diagnostic groups (n) & Hepatic ischaemia (68) & Pancreatobiliary (33) & Hepatocellular (23) & Malignancy/other (6) \\
\hline Subdivision of causes (n) & Sepsis (32) & Cholangitis (13) & Paracetamol (9) & Liver secondaries (4) \\
& CCF (17) & Pancreatitis (9) & AIH (4) & Hepatoma (1) \\
& Hypoxia (11) & CBD stone (5) & Ecstasy (3) (3) & Hepatic haematoma (1) \\
& Hypotension (8) & Pancreatic cancer (4) & Hepatitis B (3) & EBV (2) (2) \\
& & Blocked stent (1) & Alcohol (2) & \\
\hline
\end{tabular}

CCF, congestive cardiac failure; CBD, common bile duct; AIH, autoimmune hepatitis; EBV, Epstein-Barr virus.

Swansea as follows: City of Swansea (post codes SA1-SA9), population 226000 ; part of the surrounding rural area (post codes SA14, 15 , and 18), population 25000 . This population is $51.9 \%$ female and is predominantly of European ethnic origin (98.5\%). ONS figures for 1990-1994 show a $0.2 \%$ population growth with little migration or immigration.

The biochemistry department informed one of the clinicians involved in the study of the whereabouts of patients with AST greater than $400 \mathrm{U} / 1$ on a daily basis. If the patient was hospital based the clinical record was examined, or if in the community the general practitioner was telephoned and the case discussed. The following details were obtained: age, sex, hospital number, hospital speciality and full biochemical profile, relevant medical history, findings on examination, and results of special investigations. The clinical records were examined to determine whether the raised AST had been noted by the clinicians in charge and if so whether the cause of this elevation was determined. The subsequent blood tests were monitored using the notes and the pathology department computer. The final outcome following the admission was also recorded by further review of the hospital notes or by contacting the general practitioner for community cases. The final diagnosis and the pathological process responsible for the raised AST was decided by the authors on reviewing the history and investigations.

Patients were excluded from the study if there was evidence of musculoskeletal injury by trauma or ischaemia; evidence of myocardial infarction, either on ECG or history; or a raised creatine kinase.

Approval for the study was given by the local ethics committee.

\section{Results}

A total of 162 patients with an AST greater than $400 \mathrm{U} / 1$ were identified in the nine month period. Twenty five patients with raised AST of non-hepatic origin were excluded, the most common reasons being soft tissue injuries (11) and myocardial infarction (eight). Five more were excluded because of modest elevations of creatinine kinase, indicating a minor element of muscle injury superimposed on liver damage. This provided 61 men and 76 women with a median age of 66 (range 5-96 years). The median age of the men was 59 and of the women 62 years. A total of $47 \%$ of the patients $(n=64)$ were older than 70 years. All patients had other biochemical markers of liver dysfunction but not of muscle necrosis. Mean values and ranges were: bilirubin 89 (7-686)

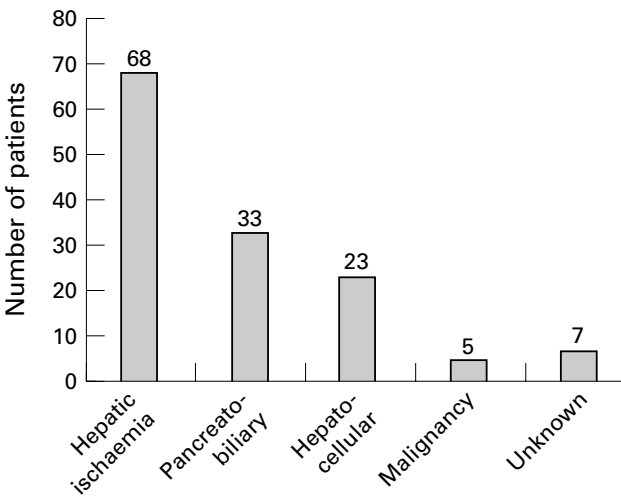

Figure 1 Causes of aspartate aminotransferase (AST) greater than $400 \mathrm{U} / \mathrm{l}$.

$\mu \mathrm{mol} / \mathrm{l}$; alkaline phosphatase 277 (40-1685) $\mathrm{U} / 1 ; \gamma$ glutamyltransferase 324 (18-2304) U/1; and creatine kinase 124 (55-220) U/1. Liver biopsies were performed in seven patients: three with autoimmune hepatitis, two with alcoholic hepatitis and cirrhosis, and one with Ecstasy induced liver injury.

All the patients identified were admitted to hospital, although in eight the index blood sample was taken in the community by the general practitioner. Ninety five were initially admitted to a medical ward and of these two were on a coronary care unit, but none had any evidence of myocardial infarction. Twenty eight patients were on surgical wards, and 14 were on an intensive care unit. Figure 1 and table 1 show the final diagnosis reached for the patients with a raised AST.

HEPATIC ISCHAEMIA AND HYPOXIA

There were 68 patients with conditions producing a decrease in hepatic blood flow or oxygenation causing hepatic ischaemia or hypoxia (fig 2). The most common cause of hepatic ischaemia (32 patients) was non-biliary sepsis. In 12 patients sepsis was caused by pneumonia; in eight it was caused by peritonitis; four patients were undergoing haemodialysis; three patients with lymphoma were undergoing chemotherapy; in two it was caused by cellulitis; in one patient there was a dental abscess; in one a urinary tract infection; and in one septicaemia postoperatively. Seventeen patients had congestive cardiac failure leading to hepatic ischaemia; all had normal creatine kinase and no evidence of myocardial infarction on ECG. There were 11 patients with hypoxia, one following a suicide attempt with asphyxia and the rest with ventilatory failure; $\mathrm{PaO}_{2}$ ranged from 4.8 to $10 \mathrm{Kpa}$ (mean 7.5). Eight patients had hypotension: systolic pressure 65-105 mm 


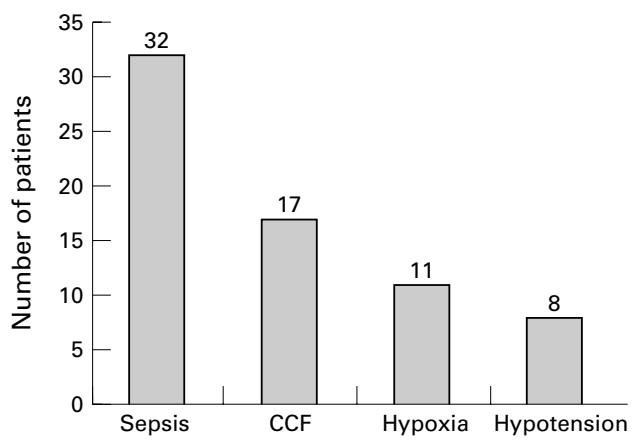

Figure 2 Aetiology of hepatic ischaemia.

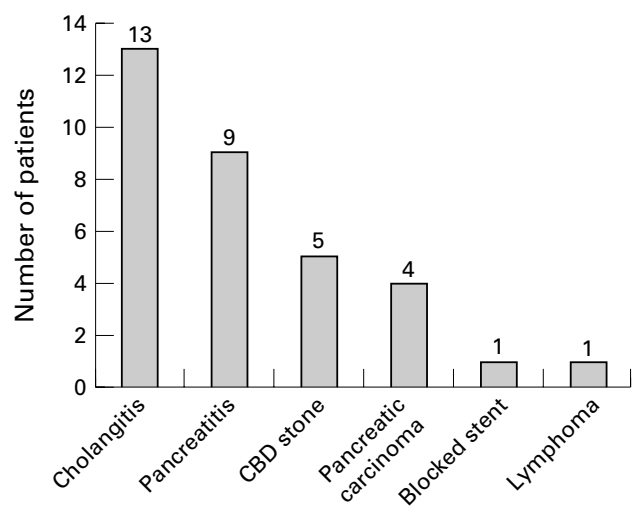

Figure 3 Subdivision of pancreatobiliary causes of aspartate aminotransferase (AST) greater than $400 \mathrm{U} / \mathrm{l}$.

$\mathrm{Hg}$ (mean 70). Two presented with a large gastrointestinal haemorrhage, two had operations with prolonged hypotension, two had arrhythmias as the cause, and two had other causes of hypotension. Many patients had several of these factors operating in concert, and in those with sepsis, 12 (38\%) had associated hypotension.

PANCREATOBILIARY DISEASE

In 33 patients the raised AST was caused by pancreatobiliary disease (fig 3). Thirteen patients had cholangitis and this diagnosis was made when there were signs of sepsis in the presence of abnormal bile ducts on ultrasonography or on endoscopic retrograde cholangiopancreatography (ERCP). There were nine patients with acute pancreatitis, all with amylase values greater than $900 \mathrm{U} / 1$ and abdominal pain; of these, eight had gall stones on ultrasonography. Four patients had bile duct obstruction caused by carcinoma of the pancreas. One patient had a blocked bile duct stent, which had been inserted for a stricture caused by chronic pancreatitis. There were five patients who had common bile duct stones without evidence of infection. One patient had lymphoma with lymphadenopathy around the porta hepatis obstructing the common bile duct. The range of systolic pressure was 100-180 mm Hg (mean 138).

PRIMARY HEPATOCELLULAR DISEASE

Twenty three patients had hepatocellular disease caused by hepatitis or damage by toxins (drugs/alcohol; fig 4). Five patients had acute viral hepatitis: three caused by hepatitis $B$ and

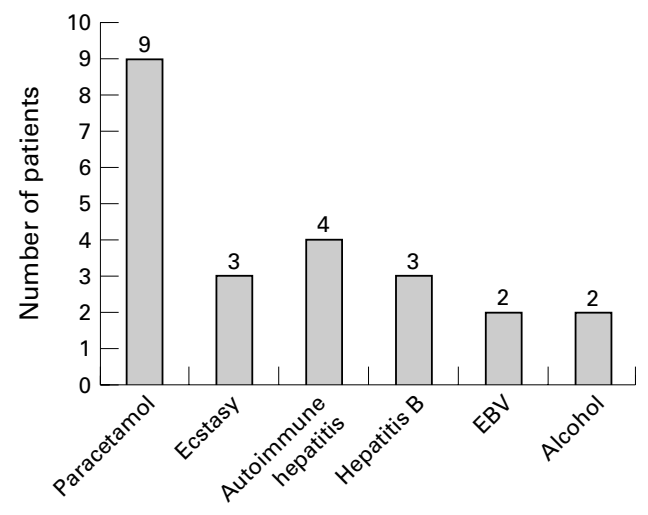

Figure 4 Hepatocellular causes of aspartate aminotransferase (AST) greater than $400 \mathrm{U} / \mathrm{l}$.

two by infectious mononucleosis. Twelve patients had drug induced liver damage: nine by paracetamol and three by Ecstasy. Two patients had alcoholic hepatitis. Four patients had autoimmune hepatitis.

\section{MALIGNANCY AND MISCELLANEOUS}

Five patients were found to have a hepatic malignancy; of these, four had metastatic disease - two from carcinoma of the breast, one from carcinoma of the stomach, and one of unknown origin - and there was one hepatocellular carcinoma. One patient had a hepatic haematoma.

\section{DIAGNOSIS UNCERTAIN}

There were seven patients (median age 59 years, five women), where the diagnosis was unclear in spite of full re-evaluation of all the clinical information and investigations in the case notes. One had taken antibiotics a week prior to admission, four were possibly related to alcohol, one had possible biliary colic with a normal ultrasound scan, and one had epigastric pain with normal amylase and normal ERCP. All the patients were normotensive throughout.

BIOCHEMICAL AND AGE DIFFERENCES

The peak AST concentrations varied widely but there was no statistical difference between diagnostic groups (see fig 5). Hepatic ischaemia had a median AST of $843 \mathrm{U} / 1$ with a range of 406-7759 U/1. Pancreatobiliary disease had a median of $625 \mathrm{U} / 1$ with a range of 405-2936 $\mathrm{U} / 1$. Hepatocellular disease had a wider range, from 401 to $27577 \mathrm{U} / 1$; the highest concentration was in a patient who had taken a paracetamol overdose. The median was however similar to the other groups at $961 \mathrm{U} / 1$.

The median age of the patients was 66 years but there were clear age differences between the diagnostic groups. The patients with a drug induced liver damage or viral hepatitis were young (median 25 years). Pancreatobiliary causes were most common in the 40-60 year age range (median 56 years), while those with ischaemic liver damage were older (median 75 years). 


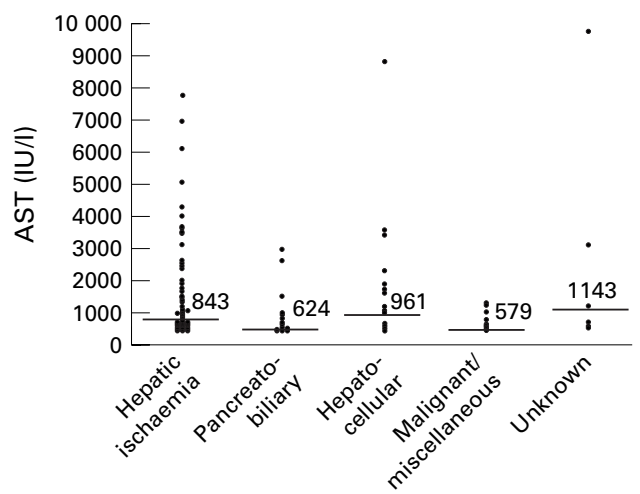

Figure 5 Peak aspartate aminotransferase (AST) values for each final diagnosis (with medians); two points excluded: 27575 (hepatocellular) and 10393 (unknown).

OUTCOME

The notes of the patients in the study were kept under review and the clinical and biochemical outcome determined. Death in hospital or discharge home and the time taken for AST to fall below $80 \mathrm{U} / 1$ (twice normal) were recorded. This AST concentration was chosen to provide sufficient numbers for meaningful analysis.

Twenty two per cent of the patients $(30 / 137)$ in the study died during their hospital admission. The mortality was highest in the hepatic ischaemia group $(37 \% ; 25 / 68)$. Two of the four patients with carcinoma of the pancreas died within two weeks of the peak AST result.

Among the 23 patients with primary hepatocellular disease there were no deaths. Of the five patients with primary or secondary tumours of the liver three died within two weeks of the peak AST.

Biochemical follow up data sufficient to calculate the duration of recorded elevation of AST were available from 70/137 patients. In the remainder insufficient data were available either because of death $(n=34)$ or because blood tests were not repeated $(n=35)$. In the hepatic ischaemia group 32 patients had complete biochemical follow up; the median time for AST to fall below $80 \mathrm{U} / 1$ was seven days (SD 5.6). In the pancreatobiliary group complete data were available in 21 and the median time for fall in AST was five days (SD 11.8). There was no statistical difference between these groups by Student's $t$ test. In the primary hepatocellular group (14 data sets) there was a significant prolongation of AST elevation of 50 days (SD 12.3), mainly accounted for by patients with "true" hepatitis (autoimmune).

The peak AST concentrations were compared with peak concentrations of bilirubin and urea and the lowest concentrations of sodium, as these biochemical parameters were frequently abnormal in the patients under study. However, no correlation with sodium, bilirubin, or creatinine was found in any group and there was only a weak correlation with urea in the hepatic ischaemia and pancreatobiliary groups.

The final aim of the study was to determine how many patients had a correct interpretation of the liver function tests recorded in the notes. This was found in 48\% (66 patients). In 14\%
(19 patients) the raised AST was noted but either an incorrect diagnosis or no interpretation was made. In 38\% (52 patients) the raised AST was apparently not noticed as no comment was made in the notes.

\section{Discussion}

Textbooks of hepatology state that AST values are a sensitive indicator of hepatocyte damage and are thus particularly useful in the early detection of viral hepatitis and in monitoring its progress. In a standard American text, Schiff's Diseases of the liver, Kaplan ${ }^{9}$ gives the commonly held view that AST concentrations exceeding 300-400 U/1 usually indicate acute hepatocellular disease such as hepatitis or drug toxicity, and points out that similar values may sometimes occur with extrahepatic obstruction but are uncommon. Kaplan ${ }^{9}$ also comments that the greatest elevations (greater than 1000 $\mathrm{U} / \mathrm{l}$ ) are seen in the early phase of viral hepatitis, in toxic injury, and with circulatory collapse, but the last of these is not considered a common cause. In an important British study ${ }^{4}$ on serum enzyme concentrations in diseases of the liver and biliary tree involving over 1100 patients, circulatory collapse did not feature at all. In a major European reference work, the Oxford Textbook of Clinical Hepatology, ${ }^{5}$ it is stated that AST concentrations greater than 20 times normal are strongly suggestive of viral or drug hepatitis but it is noted that similar concentrations may sometimes be seen in shock and heart failure, usually with a very abrupt rise. The authors also remark on the occasional striking elevation of AST in extrahepatic biliary obstruction which may mimic hepatitis. All liver textbooks and monographs on interpretation of laboratory tests agree that AST elevations below $400 \mathrm{U} / 1$ are seen in a very wide range of hepatobiliary disorders and do not help in differential diagnosis. ${ }^{5} 7910$

Our study paints a very different picture to that portrayed in the standard texts. Hepatitis, viral or otherwise, is a distinctly uncommon cause of a typical "hepatitic" biochemical result in this part of the world; furthermore, drug toxicity is not very common. Only $3.5 \%$ of our study patients had viral hepatitis and $8.5 \%$ drug toxicity as a cause of their blood picture. Ischaemic or anoxic liver damage $(50 \%)$ and extrahepatic biliary obstruction $(24 \%)$ on the other hand were far and away the most common causes. Why should there be such a major disparity between textbook descriptions and actual clinical practice? We believe there are several factors at play:

- Acute viral hepatitis is now a rare disease in South Wales ${ }^{11}$ and probably in much of the United Kingdom away from inner city areas with high risk populations.

- The elevations of AST in biliary obstruction and shock are often transient and may be missed without frequent monitoring. Received wisdom on interpretation of LFT in textbooks is based on studies from previous decades when full biochemical profiles were less commonly performed, particularly in patients without obvious liver disease; thus current practice picks up abnormal LFT 
which in the past remained undetected. Studies on LFT and their interpretation mostly come from tertiary centres of special expertise in liver disease which would largely exclude elderly patients with shock and many with acute transient biliary obstruction.

In our study the overall mortality in patients with a notably raised AST was high but all the deaths occurred in those patients with hepatic ischaemia or malignancy. Thus very high AST concentrations are associated with a very poor prognosis in patients with sepsis, congestive cardiac failure, or shock. Despite the potential value of this information we found that clinicians often miss or ignore the results of abnormal LFT and only infrequently record the correct interpretation. The reasons for this have to be speculative but it may be that abnormalities of LFT which do not fit preconceived patterns of disease are more readily disregarded by clinicians without a special interest in digestive disorders.

To have had more recorded values of AST would have helped in determining the causes of liver damage; however, the study design was such that the investigators took no part in clinical management of the patients under study. After the results of this study were presented at local audit meetings we believe that clinicians in our hospitals became more aware of the significance of AST elevation but we have yet to show this prospectively.

In the main we had no difficulties in categorising patients into the different diagnostic groups. Biliary obstruction, malignancy, and primary hepatocellular disease were easily definable. It was more difficult to subcategorise those patients in the group included under the heading hepatic ischaemia/hypoxia. We tried to subdivide this into sepsis, cardiac failure, hypoxia, and hypotension but many patients had more than one of these factors in operation, particularly those on the intensive care unit. Where more than one mechanism was involved we have categorised the case according to what we considered the dominant mechanism.

We took care to exclude patients with clinical or biochemical evidence of skeletal or myocardial damage but we accept that AST is not specific to the liver. To be included in this study all patients had to have other biochemical evidence of hepatic dysfunction so we believe that the raised AST did have a hepatic origin. (ALT would have been a rather more specific marker for liver damage but it is not part of the standard biochemical screen in our laboratory.) Liver biopsies were performed in only seven cases and all were abnormal.
It is of some interest that although alcohol is the most common cause of primary hepatocellular disease in this community, ${ }^{11}$ in this study only two patients had a notable elevation of AST caused by alcohol and in neither case did the AST concentration exceed $600 \mathrm{U} / 1$.

Both of these patients had a liver biopsy confirming cirrhosis. The first who had a peak AST of $482 \mathrm{U} / 1$ showed mixed macro- and micronodular cirrhosis. The second with peak AST of $500 \mathrm{U} / 1$ showed established cirrhosis with fatty infiltration and piecemeal necrosis. Previous studies have also shown that AST does not generally rise very high in alcoholic liver disease, whatever its severity. ${ }^{12}$ It is important to note that during the nine months of this study no patients were found to have hepatitis A as a cause of their enzyme elevation and indeed during this time no cases were recorded by the public health laboratory serving the same population (Dr A Lewis, personal communication).

In conclusion, we believe that standard teaching and hepatology textbooks offer an interpretation of LFT which does not accord with clinical practice in a district hospital in South Wales. We have found that liver anoxia and calculous biliary obstruction are the common causes of a hepatitic biochemical picture while viral hepatitis and drug hepatitis are comparatively rare.

This work has been published in part in abstract form (Hawkes ND, Whitehead M, Hainsworth I, Kingham JGC. Interpretation of a hepatitic biochemical picture. Gut 1997;40(suppl 1):F248).

1 Zilva JF, Pannall PR, Mayne DM. Clinical chemistry in diagnosis and management. London: Edward Arnold, 1988.

2 De Ritis F, Coltorti M. Attivita transaminasica del siero umano nell 'epatita virale. Minerva Med 1955;46:1207.

3 Wroblewski F, La Due JS. Serum glutamic oxalacetic transaminase activity as an index of liver cell injury: a preliminary report. Ann Int Med 1955;43:345-60.

4 Ellis G, Goldberg DM, Spooner RJ, et al. Serum enzyme tests in diseases of the liver and biliary tree. Am $\mathcal{F}$ Clin Pathol 1978;70:248-58.

5 McIntyre N, Rosalki S. Biochemical investigations in the management of liver disease. In: McIntyre N, Benhamou JP, Bircher J, et al, eds. Oxford textbook of clinical hepatology. Oxford: Oxford University Press, 1991:293-309.

6 Ginsberg AL. Very high levels of SGOT and LDH in patients with extrahepatic biliary tract obstruction. Am $\mathcal{F}$ Dig Dis 1970;15:803-7.

7 Reichling JJ, Kaplan MM. Clinical use of serum enzymes in liver disease. Dig Dis Sci 1988;33:1601-14.

8 International Federation of Clinical Chemistry, Committee on Standards, Enzyme Panel. Clin Chem 1978;24:720-1.

9 Kaplan MM. Laboratory tests. In: Schiff L, ed. Diseases of the liver. Philadelphia: JB Lippincott, 1993:108-44.

10 Moseley RH. Evaluation of abnormal liver function tests. Med Clin North Am 1996;80:887-906.

11 Kingham JGC. Clinical hepatology: alcoholic liver disease, not viral hepatitis predominates in South Wales. Hepatology 1997;25:1297.

12 Cohen JA, Kaplan MM. The SGOT/SGPT ratio-an indicator of alcoholic liver disease. Dig Dis Sci 1979;24:835-8. 